\title{
PREDICTION MODEL FOR THE CHARACTERISTICS OF NON-SPECULAR WAVE SCATTERED FROM BUILDING SURFACE
}

\author{
Hary Budiarto, Katsuyuki Haneda and Jun-ichi Takada* \\ Graduate School of Science and Engineering, Tokyo Institute of Tech. Japan
}

\section{Introduction}

The wave propagation prediction models are very crucial in determining propagation characteristics for any arbitrary installation on the implementation of mobile radio communication system. The prediction models are required for a proper coverage planning and the determination of multipath effects as well as for interference. However, reflection, diffraction, and scattering on the building surfaces in the radio environment induce undesirable multipath propagation [3].

In the preceding research [1][2] show that multipath propagation can be observed at many scatterers on the building surface roughness. The non-specular of arrival waves from building surface are more dominated by vertical and horizontal frames of window. The diffracted waves that correspond to Keller's law of diffraction can be investigated. The most of arrival waves have a tendency to be distributed around the angle of specular direction. However, more detail the scientific reasons to understand the propagation phenomena are still required.

This paper presents the development of simulation techniques for the estimation of non specular wave propagation characteristics on the building surface. Physical Optics (PO) approximation is performed to approximate equivalent currents and the total fields on the integration surface. Model of the rectangular microstrip array antenna was scanned spatially to detect multipath wave scattering. Superresolution method was also applied as an approach to handle the signal parameters (DOA, TOA) of the individual incoming waves scattered from building surface roughness. The experimental and simulation results of signal parameter of arrival waves are compared in order to investigate accuracy of the prediction model.

\section{Environment Under Consideration}

According propagation measurement in the experiment, rectangular single and array microstrip antennas are applied in the transmitter and receiver antennas model, respectively. Patch size of the antenna model was $0.0179 \times 0.0179 \mathrm{~m}^{2}$ on a dielectric substrate with $\varepsilon_{\mathrm{r}}=2.55$. The frequency of the antennas was 4.85 to $5.05 \mathrm{GHz}$. The wavelength was comparable with or smaller than depth of building surface roughness. The spatial scanning was configured to resemble an array antenna in the receiver antenna model. The spatial scanning was discretized for every $0.025 \mathrm{~m}$ toward the horizontal and vertical directions. Observation point of the simulation was performed along $0.5 \mathrm{~m}$ in the vertical and $8.125 \mathrm{~m}$ in the horizontal direction. The transmitter antenna is positioned facing towards the 
bricks' surface of the building. The profile of the building surface is shown in Fig. 1. The profile was taken from one of the buildings at Tokyo Institute of Technology. The surface of the building has non-uniformity due to the windows (glass), frames (aluminum), and walls (bricks).

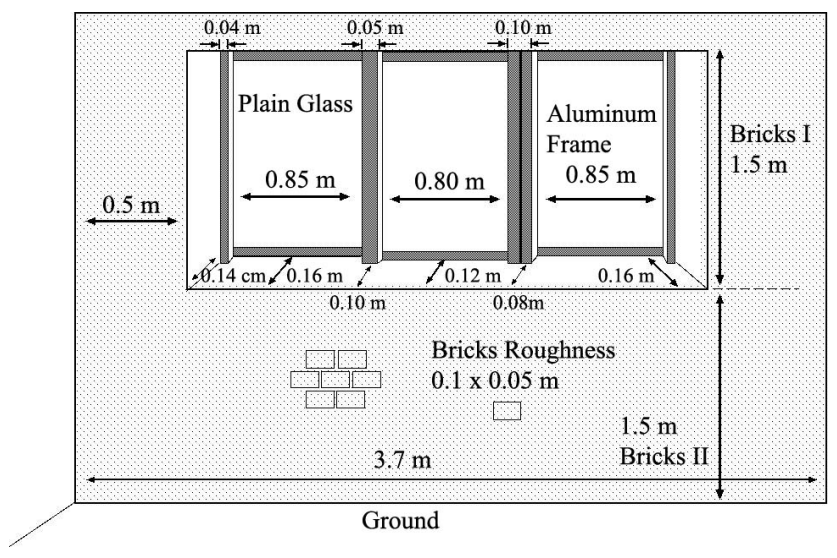

Figure 1. Building Surface Profile

\section{Physical Optics Current on Impedance Surface}

According to the field equivalence principle, equivalent currents on the illuminated part of the surface with impedance $Z[\Omega]$ are approximated by Eq. (1) and (2) in the sense of Physical Optics (PO), where non-uniform terms are neglected. $\mathbf{E}^{\text {inc }}$ and $\mathbf{H}^{\text {inc }}$ are the incident electric and magnetic at the integration point, respectively. The parameter $\alpha$ is related to the reflection coefficients of the scatterer and is expressed as Eq.(3), where $\eta=\sqrt{\mu_{0} / \varepsilon_{0}}[\Omega]$ and $Z=\sqrt{\mu / \varepsilon} \quad[\Omega]$ shows the intrinsic wave impedance of free pace and of medium, respectively.

$$
\begin{aligned}
& I_{P O}=(1+\alpha) n \times H^{i n c} \\
& M_{P O}=(1-\alpha) E^{i n c} \times n \\
& \alpha=\frac{\eta-Z}{\eta+Z}
\end{aligned}
$$

\section{Simulation Result}

The prediction model to estimate the wave scattering on the building's surface consists of four parts. The first part is for calculating radiation pattern or far field pattern from the transmitter antenna model. The second part is for obtaining the equivalent current on the building surface that has been discretized into smaller elements. The third part is for calculating total field of the receiver antenna. Lastly, the fourth part is for getting multipath signal using superresolution technique (ESPRIT). The arrival wave analyses were performed at 60 observation points with an interval of $12.5 \mathrm{~cm}$.

Figure 2 shows the ESPRIT results for azimuthal angle of the arrival wave. The azimuthal angle is defined, hence Line of Sight (LOS) is $-90^{\circ}$. The number 
of icons on the vertical line corresponds to the number of multipath signals. The figure also shows that most of the azimuthal angle values have a tendency to be distributed arround the angle of specular direction. This reason is in good agreement with experimental investigation [1] [2]. The diffracted waves from vertical and horizontal window frames are investigated in a few of scattering point at 2 to 5 meter distance between transmitter and receiver antenna. Unlike experiment result, the diffracted wave that correspond to Keller's law of diffraction and second order scattering can not be investigated.

Figure 3 shows elevation angle of the arrival wave. It can be seen that the value of elevation angle is around $0^{0}$ for those arrival waves coming from windows and bricks-I scatterers. It implies that most of the scattered waves from building surface arrive closely from specular directions. The arrival wave from Bricks II scatterer can be observed in the figures. The diffracted wave from vertical and horizontal window frames are investigated in a few scattering points.

Figures 4 shows path gain and delay time of arrival wave from windows scatterer. The path gain values of arrival wave are compared between with experiment and simulation result. A good agreement is indicated between experiment and simulation result for path gain of the reflected wave. The figures also show that delay time directly estimated by ESPRIT yields close agreement with delay time estimated by using propagation path based on free space velocity of $3 \times 10^{8} \mathrm{~m} / \mathrm{s}$. Based on these result, the signal parameter of experimental result and of simulation result have a little difference. The difference is attributed to the accuracy of element discretized in the roughness of building surface.

\section{Conclusion}

This paper presents propagation prediction model of non-specular wave scattering from building surface. The calculation method to calculate the total field of the receiver antenna consists of four parts: calculate the far field pattern of the transmitter antenna, determine the equivalent current on the building surface, calculate the total field on the receiver antenna, and finally obtain the multipath signal by applying ESPRIT. Numerical results show that the signal parameter profile is in good agreement with the experimental results. However, there are observed a little difference between the numerical and experimental result. The difference is attributed to the accuration of element discretized in the roughness of building surface.

\section{Reference}

1. H. Budiarto, K. Horihata, K. Haneda, and J. Takada, "Polarimetric Measurement of Nonspecular Wave Scattering from Building Surface Roughness", IEEE Antenna and Wireless Propagation Letters, Volume 2, Issue 16, pp. 242-245, 2003.

2. H. Budiarto, K. Horihata, K. Haneda, and J. Takada, "Superresolution Measurement of Nonspecular Wave Scattering from Building Surface Roughness", Proc. of IEEE VTC Fall 2003 Conf., 07A_03, October 2003, Orlando-USA.

3. Takada, J. Fu, H. Zhu, and T. Kobayashi, "Spatio-Temporal Channel Characterization in a Sub-Urban Non-Line-of-Sight Microcellular Environment", IEEE J. Select. Areas in Comm., vol. 20, no. 3, pp.532-538, Apr. 2002.

4. K. Sakaguchi, J. Takada and K. Araki, "A Novel Architecture for MIMO Spatio-Temporal Channel Sounder," IEICE Trans. on Electronics, vol. E-86C, no. 3, pp. 436-441, Mar. 2002. 


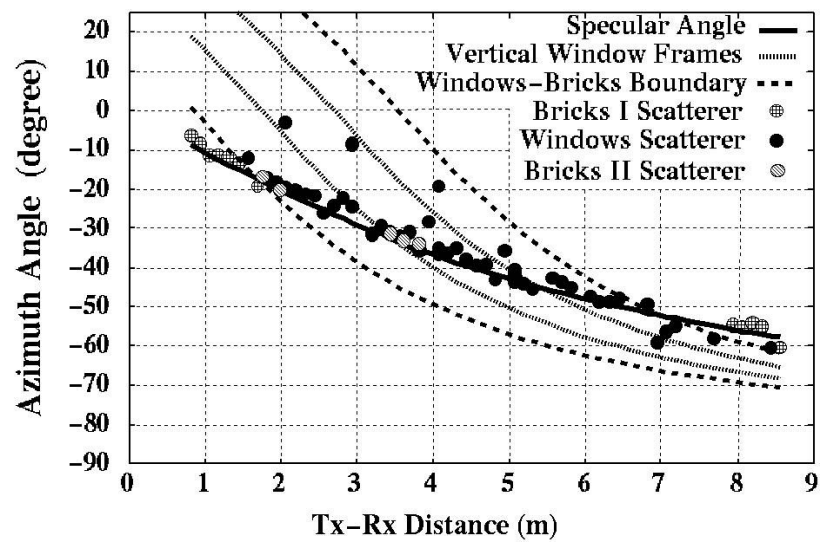

Figure 2. Azimuth Angle of Arrival Wave for Prediction Model

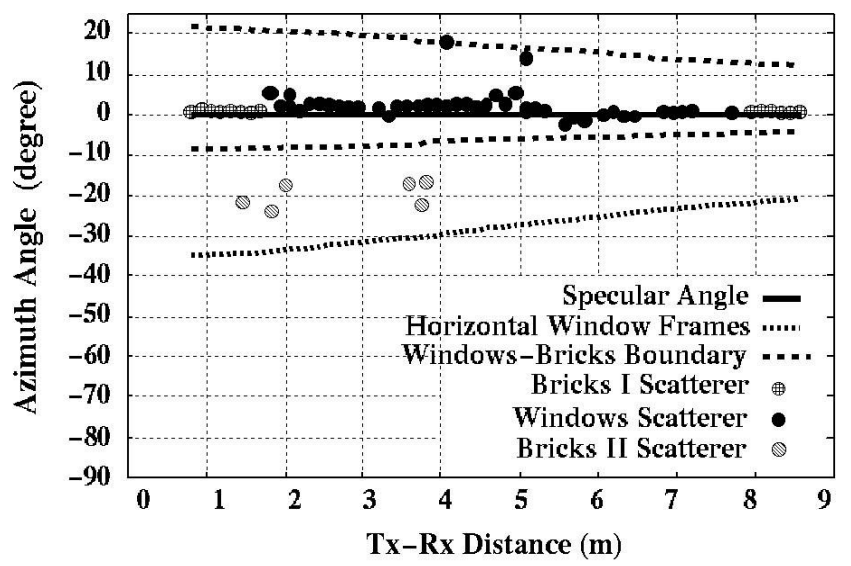

Figure 3. Elevation Angle of Arrival Wave for Prediction Model

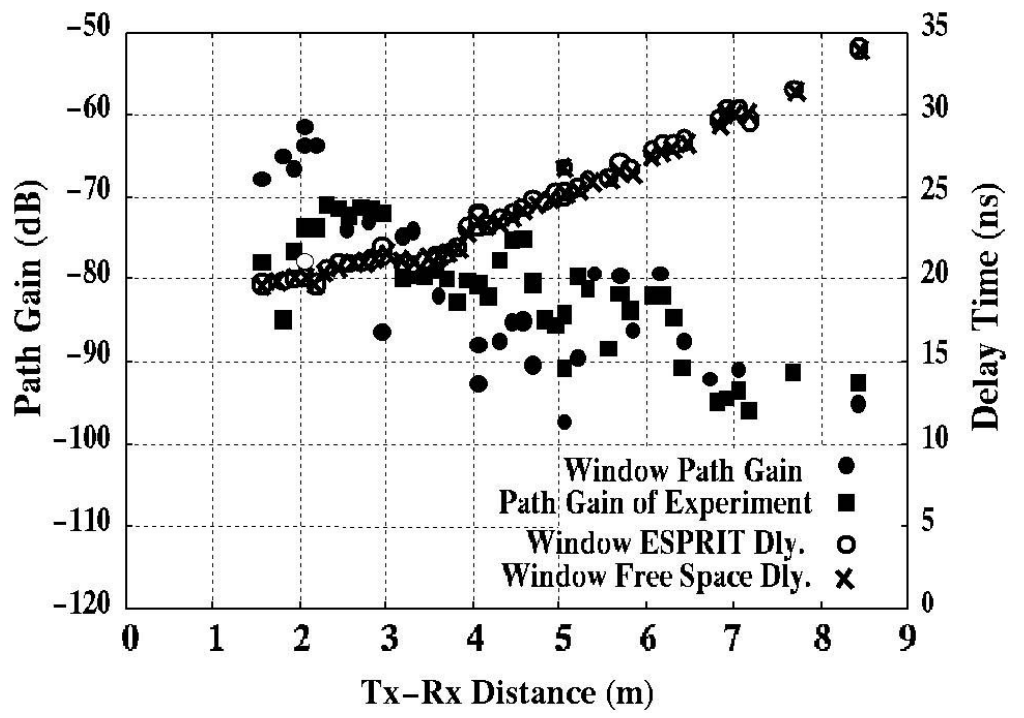

Figure 4. Path Gain and Delay Profile of Arrival Wave from Windows Scatterer 